\title{
Electrosmelting of Lead-Containing Dusts from Copper Smelters
}

\author{
G. Z. Moldabayeva ${ }^{1}$ - S. K. Akilbekova ${ }^{1}$ K. K. Mamyrbayeva ${ }^{1} \cdot$ B. Mishra $^{2}$
}

Published online: 28 August 2015

(C) The Minerals, Metals \& Materials Society (TMS) 2015

\begin{abstract}
Experimental and mathematical modeling work has been conducted to develop an effective technological scheme for processing lead-containing dusts generated in copper smelting. The work incorporates conditions of melting, charge composition, and the physical parameters of the dust. The optimal conditions of electrosmelting have been established for complete and comprehensive extraction of the basic and rare metals from the dust. In this onestage processing, three intermediate products have been recovered: crude lead; basic matte-slag melt containing $\mathrm{Cu}$, $\mathrm{Zn}$, and As; and secondary sublimates enriched in $\mathrm{Cd}$ and Re.
\end{abstract}

Keywords Lead-containing dust - Copper smelting · Electro-thermal processing · Rough lead · Basic matte-slag melt $\cdot$ Rhenium $\cdot$ Secondary sublimates $\cdot$ Mathematical modeling

The contributing editor for this article was K. Yamaguchi.

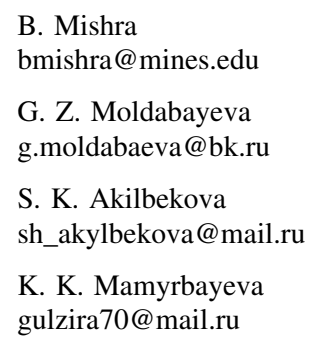

1 Kazakh National Technical University Named After K.I. Satpayev, Almaty, Kazakhstan

2 Colorado School of Mines, Golden, CO, USA

\section{Introduction}

A significant number of diverse compositions of dry dusts and sublimates are obtained during the roasting, sintering, sulfide smelting, converting of copper and copper-lead mattes from copper production plants. For example, dusts of copper-smelters contain valuable metals, such as $\mathrm{Pb}, \mathrm{Zn}$, $\mathrm{Cu}, \mathrm{Cd}, \mathrm{Bi}, \mathrm{Sb}, \mathrm{Re}, \mathrm{Te}, \mathrm{Se}$, and Tl. Due to the low content of these metals, they are essentially considered secondary concentrates. Concentrations of these and other rare dispersed elements are 4-10 times higher than in the initial mined raw materials [1].

Since accumulation of dusts in these processes is an unavoidable problem and is generally considered metallurgical waste, development of new technological methods for their complex processing is required to achieve complete extraction of base metals and rare metals. Specificity of physico-chemical properties and relatively high content of valuable components in dusts of copper smelters formed the basis for development of technological schemes of separate processing of these dusts to recover lead, zinc, cadmium, rare dispersed metals, and output of arsenic from the production.

Until now, metallurgical plants dusts have been recycled by various schemes and they usually had low extraction of basic $(\mathrm{Pb}, \mathrm{Cu}$, and $\mathrm{Zn})$ and associated metals $(\mathrm{Se}, \mathrm{Te}, \mathrm{In}$, $\mathrm{Cd}, \mathrm{Tl}$, and $\mathrm{Re}$ ). In most cases, dusts processing is carried out by hydrometallurgical method. As the leaching reagents, solutions of acids, alkalis, and sodium salts [2-4] were investigated. There are known experimental data on the two-stage leaching of the copper smelting productions dusts by sulfuric acid solutions, aqueous solutions of sodium carbonate and nitric acid, as well as with the presence of oxidizing agents [5-8]. 
Fig. 1 Experimental apparatus for electric smelting of leadcontaining dust

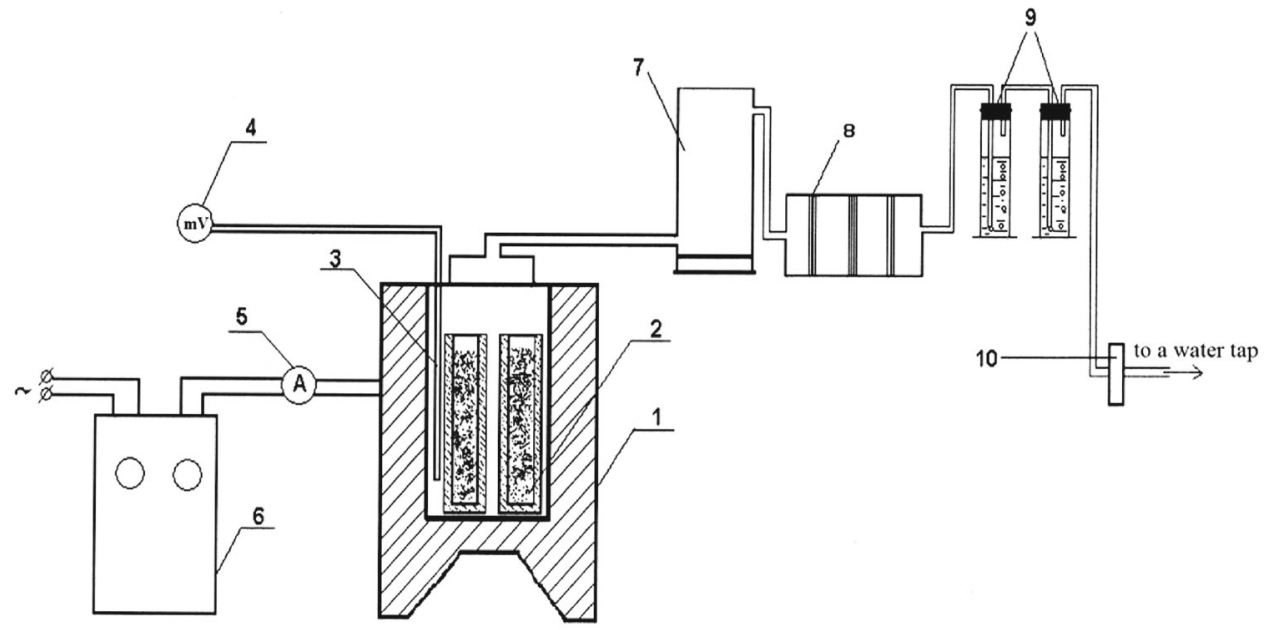

1 - shaft electric furnace; 2 - the crucible with the charge; 3 - thermocouple; 4 - millivoltmeter; 5 -ammeter; 6 - voltage regulator; 7 - dust chamber; 8 - bag filters; 9 - column for the analysis of gases and additional dust collection; 10 - water jet pump
Along with the hydrometallurgical methods of dusts processing, the pyrometallurgical processes are also considered. There is information in the literature on processing of lead-bearing dusts by roasting of specially prepared charge, a heat treatment in one or several stages and the reduction smelting in a wide range of temperatures with additives of various reagents [9-13].

Overview of the various methods of lead-bearing dusts processing of copper smelters indicates that, despite the wide range of used technology, they do not always solve the problem of complex extraction of all valuable components from this type of raw material. Most of the considered methods are characterized by a high consumption of expensive reagents, circulation of a large number of ballast returns (in the form of sulfate lead cakes), and low extraction of rare and dispersed elements. Therefore, it is necessary to find out optimal ways of complex processing of lead-containing dusts using the electrothermal combined method, which is the most promising and environmentally feasible technology [14].

\section{Experimental Methods}

As the starting materials for experiments on electrothermal recycling of lead-containing dust of copper smelters, sodium sulfate, sodium carbonate (technical soda containing 96.5-97 \% $\mathrm{Na}_{2} \mathrm{CO}_{3}$ ); as a reducing agent, charcoal and coke fines were used.

The process equipment (Fig. 1) consisting of a shaft furnace, dust collection devices, and the absorption columns for the analysis of exhausted gases was used with a semi-automatic adjustment of electrothermal melting modes with controlled electrical load and temperature. This equipment allowed the study of lead-containing dusts' electrothermal processing modes and to justify technology parameters, such as charge composition, melting temperature, and exposure duration. Weighing of starting materials was performed on electronic scales (MW-1200 with an accuracy of $\pm 0.0001 \mathrm{~g}$ ). Weighed masses of lead dusts were mixed with sodium sulfate, soda and reducing agent in different ratios according to
Table 1 Levels of investigated experimental variables

\begin{tabular}{lrrrrr}
\hline Experimental variables & \multicolumn{2}{l}{ Level } & & & \\
\cline { 2 - 6 } & \multicolumn{1}{c}{ (i) } & \multicolumn{1}{c}{ (ii) } & (iii) & (iv) & (v) \\
\hline$x_{1}$, the temperature, ${ }^{\circ} \mathrm{C}$ & 850 & 950 & 1050 & 1150 & 1250 \\
$x_{2}$, the exposure time, min. & 12 & 24 & 36 & 48 & 60 \\
$x_{3}$, the addition of $\mathrm{Na}_{2} \mathrm{CO}_{3}, \%$ of dust content & 10 & 20 & 30 & 40 & 50 \\
$x_{4}$ the addition of $\mathrm{Na}_{2} \mathrm{SO}_{4}, \%$ of dust content & 2 & 4 & 6 & 8 & 10 \\
$x_{5}$, the addition of $\mathrm{C}, \%$ of dust content & 4 & 8 & 12 & 16 & 20 \\
\hline
\end{tabular}


the goals of the study. Further, carefully intermixed charge was placed in alumina crucibles, which were put into an electric furnace [1] (Ref. Fig. 1 for identifying the equipment parts description numbers) after which the furnace temperature was raised to the required level. Control and measurement of the temperature in the reaction zone of the shaft electric furnace was performed by tungsten-rhenium thermocouple [3] and recorded with a millivoltmeter [4]. Upon reaching the desired temperature in the reaction zone, the initial charge exposure was carried out under the given time profile of 12-60 min. After completion of the experiment, crucibles were removed from the furnace, weighed, and cooled products were subjected to chemical and mineralogical analyses. During the smelting, generated sublimates were trapped in a dust chamber [7] and the filter bags [8].

Metallurgical processing of complex materials, such as dusts of copper smelters, is not only influenced by the charge composition, but also by the physical and chemical parameters of the process. In this case, identification of parameters of technological process requires complicated

Table 2 Plan-matrix of five-factor experiment

\begin{tabular}{|c|c|c|c|c|c|}
\hline \multirow[t]{2}{*}{ Test no. } & \multicolumn{5}{|c|}{ Levels of factors } \\
\hline & $x_{1}$ & $x_{2}$ & $x_{3}$ & $x_{4}$ & $x_{5}$ \\
\hline 1 & 850 & 12 & 10 & 2 & 4 \\
\hline 2 & 850 & 36 & 30 & 6 & 12 \\
\hline 3 & 850 & 24 & 20 & 4 & 8 \\
\hline 4 & 850 & 60 & 50 & 10 & 20 \\
\hline 5 & 850 & 48 & 40 & 8 & 16 \\
\hline 6 & 1050 & 12 & 30 & 4 & 20 \\
\hline 7 & 1050 & 36 & 20 & 10 & 16 \\
\hline 8 & 1050 & 24 & 50 & 8 & 4 \\
\hline 9 & 1050 & 60 & 40 & 2 & 12 \\
\hline 10 & 1050 & 48 & 10 & 6 & 8 \\
\hline 11 & 950 & 12 & 20 & 8 & 12 \\
\hline 12 & 950 & 36 & 50 & 2 & 8 \\
\hline 13 & 950 & 24 & 40 & 6 & 20 \\
\hline 14 & 950 & 60 & 10 & 4 & 16 \\
\hline 15 & 950 & 48 & 30 & 10 & 4 \\
\hline 16 & 1250 & 12 & 50 & 6 & 16 \\
\hline 17 & 1250 & 36 & 40 & 4 & 4 \\
\hline 18 & 1250 & 24 & 10 & 10 & 12 \\
\hline 19 & 1250 & 60 & 30 & 8 & 8 \\
\hline 20 & 1250 & 48 & 20 & 2 & 20 \\
\hline 21 & 1150 & 12 & 40 & 10 & 8 \\
\hline 22 & 1150 & 36 & 10 & 8 & 20 \\
\hline 23 & 1150 & 24 & 30 & 2 & 16 \\
\hline 24 & 1150 & 60 & 20 & 6 & 4 \\
\hline 25 & 1150 & 48 & 50 & 4 & 12 \\
\hline
\end{tabular}

multifactorial analysis. Staging of experiments, considering all factors would require an infinitely large number of tests. To solve this difficult problem of optimizing the number of tests, the method of mathematical modeling was employed which reduced the necessary amount of experiments for several tested parameters.

To study the processes in specific conditions, the methodology of mathematical modeling proposed by Malyshev, based on the equation of Protodjiakonov [15, 16], was used. Determination of the conditions for more complete and comprehensive extraction of heavy non-ferrous $(\mathrm{Pb}, \mathrm{Cu})$, small $(\mathrm{Cd})$, and rare $(\mathrm{Re})$ metals into the corresponding intermediates in electrosmelting of copper smelters dusts were determined according to the five-factor experimental plan. The dependence of the extraction degree from dust was studied for lead into crude lead, copper-into matte-slag melt, cadmium and rhenium - into sublimates on the experimental variables of temperature and exposure time and material variables of soda, sulfate solution, and coke additions as wt.\% of lead dust content. In accordance with the modeling matrix for the five factors, 25 independent experiments were conducted on five levels of each factor (Table 1).

Soda smelting reduction, i.e., smelting with the addition of sodium carbonate and coal/coke, provides crude metal recovery of the main component from the dust, lead, in the first stage of pyrometallurgical process. Next, black lead goes through standard refining process. The role of sodium sulfate is to melt the products and make them fusible, enabling easy black lead separation from the matte-slag melt.

The amounts of sodium sulfate, soda and coke, are added to the melt in an amount greater than the stoichiometric requirement to produce lead bullion and copper matte in the slag product, cadmium, rhenium sublimates for copper, cadmium and rhenium in sulfide form, and lead in the form of crude metal.

Analysis of the thermodynamic parameters of complex reactions, which occur during the melting of lead dust with sodium sulfate and sodium carbonate in the presence of carbon in the temperature range studied, showed that lead reduced to the metal and cadmium transferred to the sublimates. As the temperature increases, the probability of transition of lead metal and cadmium into sublimates increases. Rhenium sublimates beneficially into a heptavalent rhenium oxide and copper goes into sodium matteslag melt. It has been established that conversion to metallic lead is the most probable thermodynamic reaction when lead sulfate and oxy-sulfate react with sodium sulfate, sodium carbonate, and carbon. Gibbs free energy has a negative value even at $573 \mathrm{~K}$. With increasing temperature, the thermodynamic probability of the studied reactions also increases. 


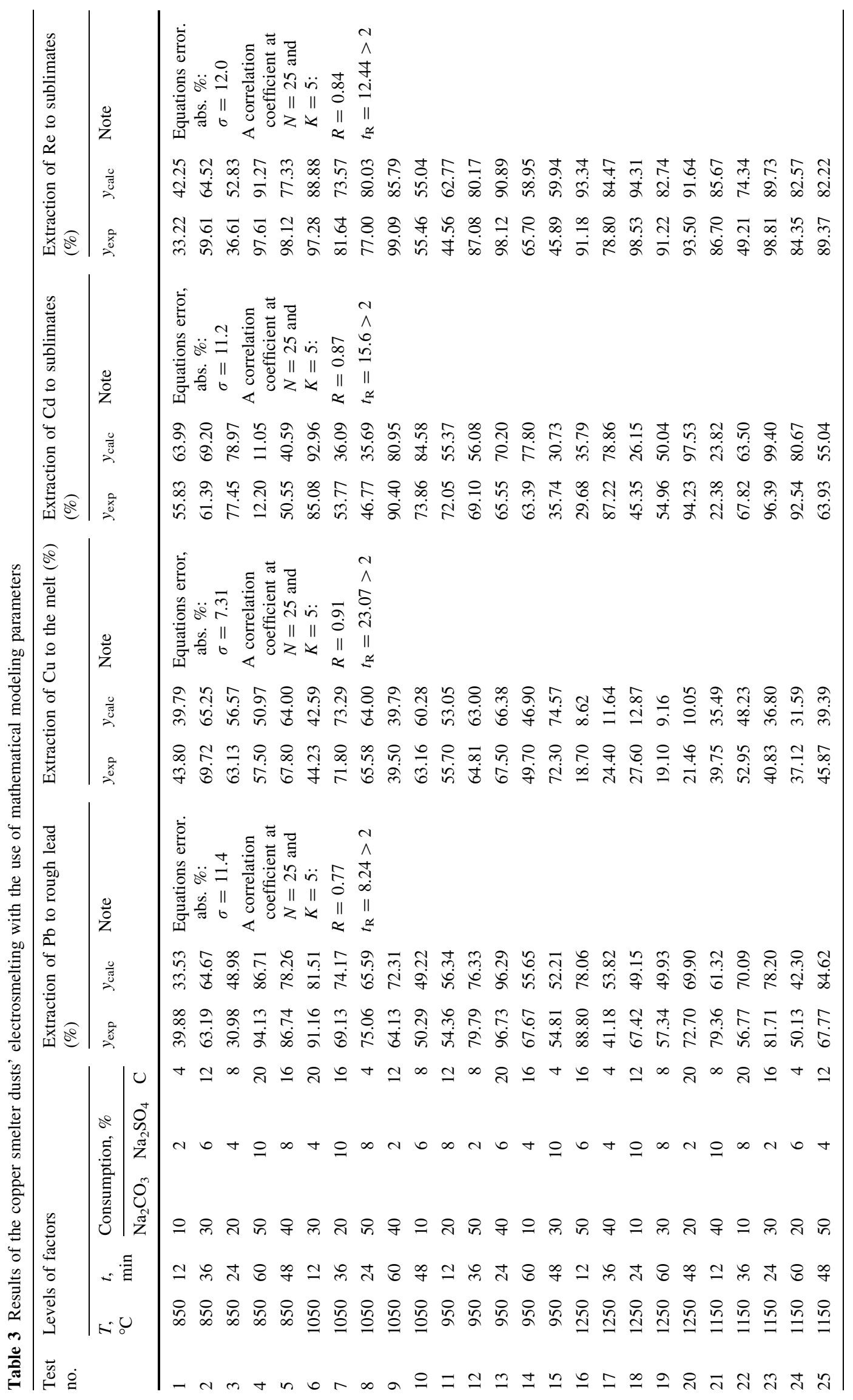


Fig. 2 Particular dependences of the extraction degrees of lead into the rough lead (1) and copper into a melt (2) on various factors
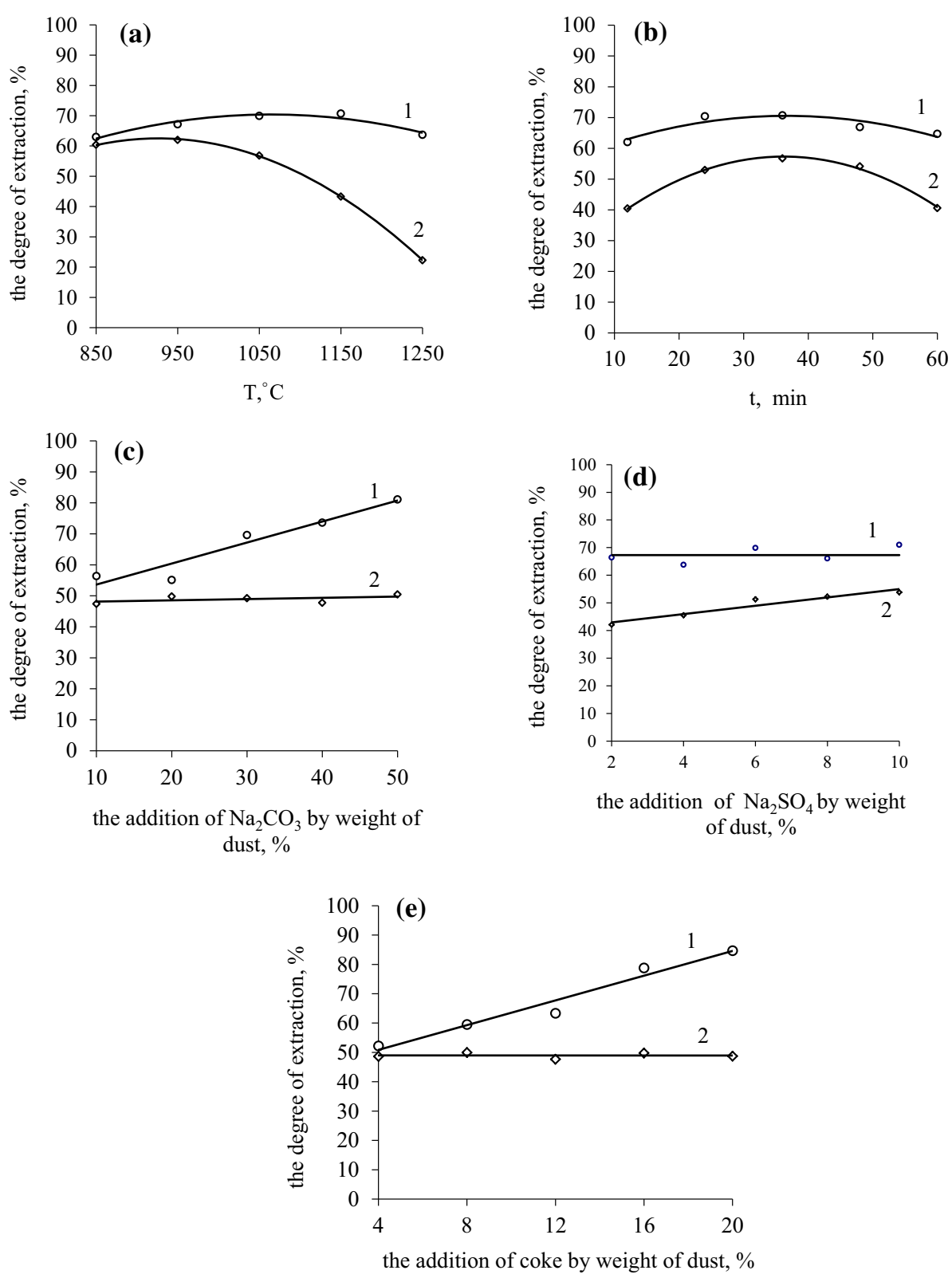

At low temperatures, formation of $\mathrm{CdO}$ is most probable thermodynamically, and the conversion to the metal cadmium as the reaction product of cadmium sulfate with soda and cadmium oxide with sodium sulfate and carbon occur.

For the formation reactions of thiosalts, copper has negative values of Gibbs energy at temperatures below $773 \mathrm{~K}$, which indicates the possibility of forming more thiosalts of $\mathrm{Cu}$ in solid phases. The thermodynamically most probable formation reaction of copper thiosalt is $\mathrm{Na}_{2} \mathrm{Cu}_{8} \mathrm{~S}_{5}$. All studied processes of the oxidation of rhenium compounds have negative values of Gibbs energy. Thermodynamically it is most likely the formation of heptavalent rhenium oxide, $\operatorname{Re}_{2} \mathrm{O}_{7}$, occurs from its sulfide.

Under laboratory conditions, the initial crucible is cooled and slag, matte-product, and lead bullion are separated by density and color. Sublimates are trapped in cyclones.

Sodium matte-slag melt is subjected to hydrometallurgical processing, followed by sulfation into cakes, as shown in Fig. 4. Aqueous leaching conditions are a temperature of $90{ }^{\circ} \mathrm{C}$ for 120 min with a stirring rate of $500 \mathrm{rpm}$. Extraction of heavy non-ferrous metals $(\mathrm{Pb}, \mathrm{Zn}$, and $\mathrm{Cu}$ ) into cake was 97.1-99.8\%, and sodium and arsenic almost completely transferred to the leach solution.

Processing of the residue cake from the aqueous leach comprised matte-slag melt with sulfuric acid at a temperature of $350{ }^{\circ} \mathrm{C}$, followed by aqueous leaching recovery of two products: lead sulfate was extended to smelting process and zinc sulfate solution rich in copper was in turn fed to electrowinning of these metals. 
Fig. 3 Particular dependences of the extraction degree of cadmium (3) and rhenium (4) into the sublimates from the investigated factors
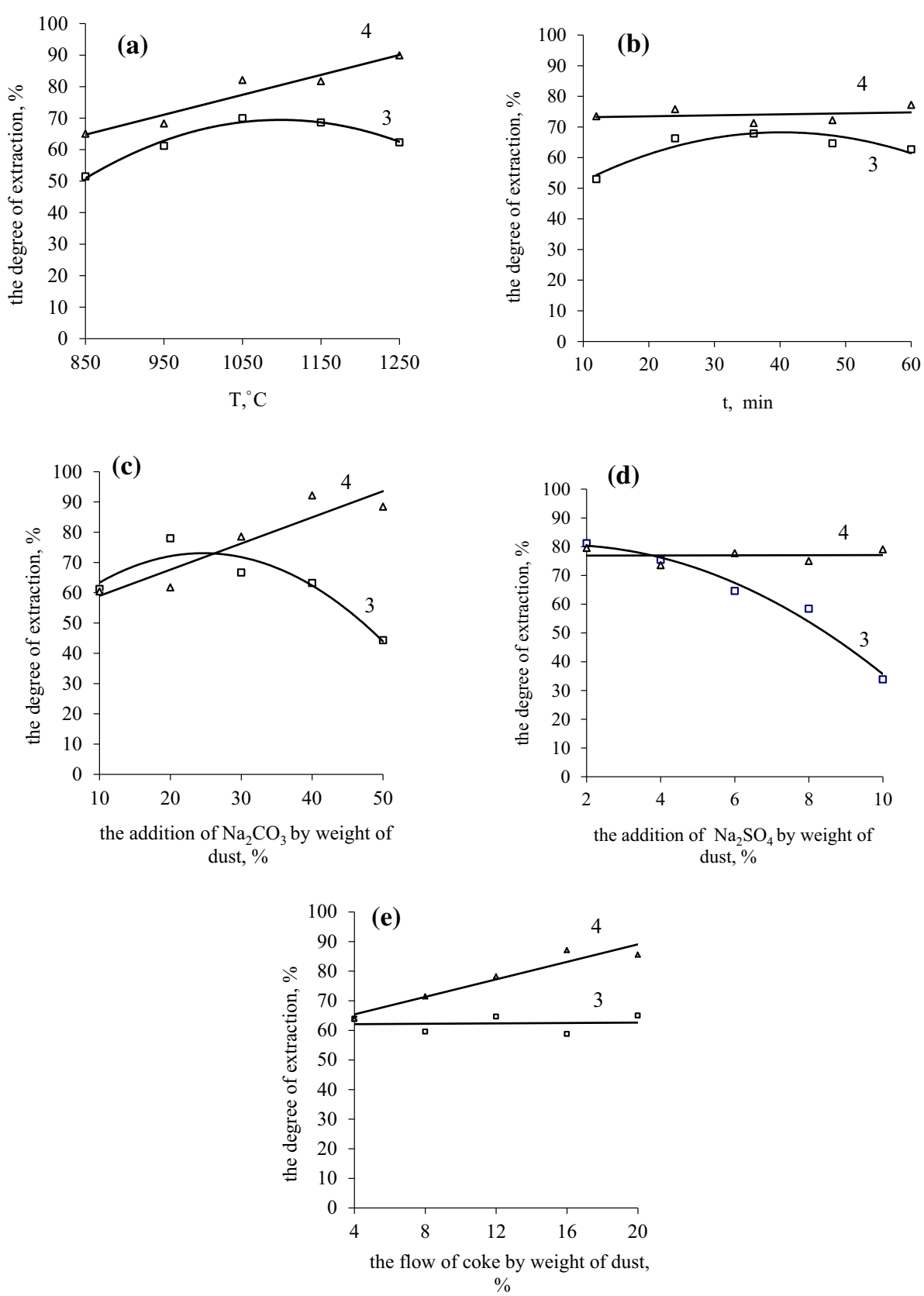

The structure of experiment plan was such that during all tests each level of any factor met once with each level of all other factors, thereby providing averaging of influence of any factor in sampling of the experimental results at any level. Experimental conditions are shown in Table 2. The parameters have to be selected based on the phase rule. The parameters are chosen as basic parameters for commodity products. Many studies conducted use not even five, but only four factorial experiments with 25 experiments. This is due to the fact that conducting 25 experiments was found to be sufficient to determine the controlling factors that affect the final results.

\section{Results and Discussion}

Based on the experimental values obtained (Table 3), graphs were plotted (Figs. 2, 3) showing particular dependency of the degree of metals extraction using the studied factors, which were approximated by the corresponding equations 
Table 4 Partial dependencies equation, correlation coefficient, and their significance factor

\begin{tabular}{|c|c|c|}
\hline Private dependence of the extraction degree & Correlation coefficient, $R$ & Correlation coefficient significance, $t_{\mathrm{R}}$ \\
\hline \multicolumn{3}{|l|}{$\mathrm{Pb}$ in black lead } \\
\hline$y_{1}=69.95-0.00022 \cdot\left(x_{1}-1050\right)^{2}$ & 0.70 & 2.382 \\
\hline$y_{2}=70.71-0.0145 \cdot\left(x_{2}-36\right)^{2}$ & 0.85 & 5.302 \\
\hline$y_{3}=44.79+0.72 \cdot x_{3}$ & 0.89 & 7.412 \\
\hline$y_{4}=61.15+0.974 \cdot x_{4}$ & 0.43 & 0.902 \\
\hline$y_{5}=42.41+2.11 \cdot x_{5}$ & 0.97 & 28.432 \\
\hline \multicolumn{3}{|l|}{$\mathrm{Cu}$ in matte-slag melt } \\
\hline$y_{1}=62-0.00057 \cdot\left(x_{1}-950\right)^{2}$ & 0.89 & 7.402 \\
\hline$y_{2}=56.74-0.03 \cdot\left(x_{2}-36\right)^{2}$ & 0.98 & 42.862 \\
\hline$y_{3}=47.75+0.0405 \cdot x_{3}$ & $\approx 0$ & $\approx 0.2$ \\
\hline$y_{4}=39.91+1.509 \cdot x_{4}$ & 0.95 & 16.882 \\
\hline$y_{5}=48.97-0.001 \cdot x_{5}$ & $\approx 0$ & $\approx 0.2$ \\
\hline \multicolumn{3}{|l|}{$\mathrm{Cd}$ in sublimates } \\
\hline$y_{1}=70.5-0.00038 \cdot\left(x_{1}-1080\right)^{2}$ & 0.94 & 13.992 \\
\hline$y_{2}=67.86-0.0197 \cdot\left(x_{2}-36\right)^{2}$ & 0.68 & 2.202 \\
\hline$y_{3}=77.0-0.0519 \cdot\left(x_{3}-23\right)^{2}$ & 0.83 & 4.622 \\
\hline$y_{4}=81.19-0.887 \cdot\left(x_{4}-2\right)^{2}$ & 0.90 & 8.202 \\
\hline$y_{5}=65.0-0.156 \cdot\left(x_{5}-12\right)^{2}$ & $\approx 0$ & $\approx 0.2$ \\
\hline \multicolumn{3}{|l|}{ Re in sublimates } \\
\hline$y_{1}=11.252+0.063 \cdot x_{1}$ & 0.95 & 16.882 \\
\hline$y_{2}=69.39+0.203 x_{2}$ & 0.096 & 0.172 \\
\hline$y_{3}=50.36+0.864 \cdot x_{3}$ & 0.90 & 8.202 \\
\hline$y_{4}=75.94+0.175 \cdot x_{4}$ & $\approx 0$ & $\approx 02$ \\
\hline$y_{5}=59.47+1.48 \cdot x_{5}$ & 0.94 & 13.992 \\
\hline
\end{tabular}

with high correlation coefficients $\left(t_{\mathrm{R}}>2\right)$. Based on the significant partial dependencies, multifactorial generalized equations for extracting degree of lead (A), copper (B), cadmium (C), and rhenium (D) were made:

$y=\frac{\left[69.95-0.00022 \cdot\left(x_{1}-1050\right)^{2}\right] \times\left[70.71-0.0145 \cdot\left(x_{2}-36\right)^{2}\right] \times\left[44.79+0.72 \cdot x_{3}\right] \times\left[42.41+2.11 \cdot x_{5}\right]}{(66.99)^{3}}$

$y=\frac{\left[62-0.00057\left(x_{1}-950\right)^{2}\right] \times\left[56.74-0.03\left(x_{2}-36\right)^{2}\right] \times\left[39.91+1.509 \cdot x_{4}\right]}{(48.96)^{2}}$

$y=\frac{\left[70.5-0.00038 \cdot\left(x_{1}-1080\right)^{2}\right] \times\left[67.86-0.0197 \cdot\left(x_{2}-36\right)^{2}\right] \times\left[77.0-0.0519 \cdot\left(x_{3}-23\right)^{2}\right] \times\left[81.19-0.887 \cdot\left(x_{4}-2\right)^{2}\right]}{(62.705)^{3}}$ 
$y=\frac{\left[11.252+0.063 \cdot x_{1}\right] \times\left[50.36+0.864 \cdot x_{3}\right] \times\left[59.47+0.48 \cdot x_{5}\right]}{(76.92)^{2}}$.

Equations error was calculated by formula (4).

$$
\sigma=\sqrt{\frac{\sum_{1}^{n}\left(y_{\exp }-y_{\text {calc }}\right)^{2}}{N-K-1}}
$$

where

$N$-number of described points;

$K$-number of acting factors;

$y_{\text {exp_experimental result; }}$

$y_{\text {calc }}$-theoretical (calculated) result.

The obtained results showed the coincidence of calculated and experimental values of the $\mathrm{Pb}, \mathrm{Cu}, \mathrm{Cd}$, and $\mathrm{Re}$ extraction degrees to the corresponding intermediates (Table 3). Despite the fact that some results of experiment and calculation differ quite significantly, none of the experimental value deviates from the calculated amount by greater than $3 \sigma$. High correlation coefficients indicate, in turn, the adequacy of generalized Eqs. 1-4 (Table 3).

Thus, studies on the combined electrothermal processing technology of copper smelter dusts with addition of soda,
$R=\sqrt{\frac{(N-1) \sum_{1}^{N}\left(Y_{\mathrm{e}}-Y_{\mathrm{t}}\right)^{2}}{(N-K-1) \sum_{1}^{N}\left(Y_{\mathrm{e}}-Y_{\mathrm{av}}\right)^{2}}}$,

where, $N$ is the number of points described, $K$ is the number of operational factors, $Y_{\mathrm{e}}$ is the experimental result, $Y_{\mathrm{t}}$ is the theoretical result, and $Y_{\mathrm{av}}$ is the average experimental value. As it can be seen from Table 4, from all partial dependencies, only the 4th was insignificant, and it is excluded from further consideration.

Next, based on the significant private dependencies, multivariate generalized equation is compiled by following formula:

$Y_{\mathrm{g}}=\frac{\prod_{i=1}^{n} Y_{\mathrm{i}}}{Y_{\mathrm{av}}^{n-1}}$

where, $Y_{\mathrm{g}}$ is the generalized function, $Y_{\mathrm{i}}$ is the dependent function. $\Pi_{i=1}^{n}$ is the multiplication of all dependent functions and $Y_{\mathrm{av}}$ is the overall average of all values considered to the extent of the distribution per unit at the amount of private functions. Thus, the degree of extraction of lead (1) is obtained by:

$y=\frac{\left[69.95-0.00022 \cdot\left(x_{1}-1050\right)\right] \times\left[70.71-0.0145 \cdot\left(x_{2}-36\right)^{2}\right] \times\left[44.79+0.72 \cdot x_{3}\right] \times\left[42.41+2.11 \cdot x_{5}\right]}{(6699)^{3}}$

sodium sulfate, and the coke to the charge, in accordance with the modeling matrix for the five factors allowed multiple correlation equations for $\mathrm{Pb}, \mathrm{Cu}, \mathrm{Cd}$, and $\mathrm{Re}$ extraction to respective products.

Initially, the curves are constructed according to the experimental values of dependent functions shown in Table 3. Next, to describe the data in Figs. 2, 3, corresponding empirical formulas are selected. For example, in lead, parabolic equation was used in the description of the partial dependencies of $y_{1}$ and $y_{2}$, while for the description of partial dependencies $y_{3}, y_{4}$, and $y_{5}$, straight line equations were used (Table 4).

Next, using a non-linear multiple correlation coefficient, significance or insignificance of the functions is determined by the following formula:
Similarly, Eqs. 2, 3, and 4 are compiled.

Melting products obtained after experiment were examined by chemical analysis method. Table 5 shows the chemical composition of black lead and sodium matte-slag melt, which were obtained after fusion. X-ray diffraction was used in, addition, only to confirm the data of chemical analysis.

The optimum extraction conditions for lead to rough lead (up to $97 \%$ ), copper to the matte-slag melt (up to $70 \%$ ), cadmium (up to $95 \%$ ), and rhenium (up to $92.2 \%$ ) into the sublimates were found. These are temperature in the range of $1100-1175{ }^{\circ} \mathrm{C}$; the duration of exposure in the range of 30-40 min; content of soda in the charge at $30-40 \%$ and sodium sulfate at $6-8 \%$ with coke requirement in the range of $10-12 \%$ by weight of dust. 


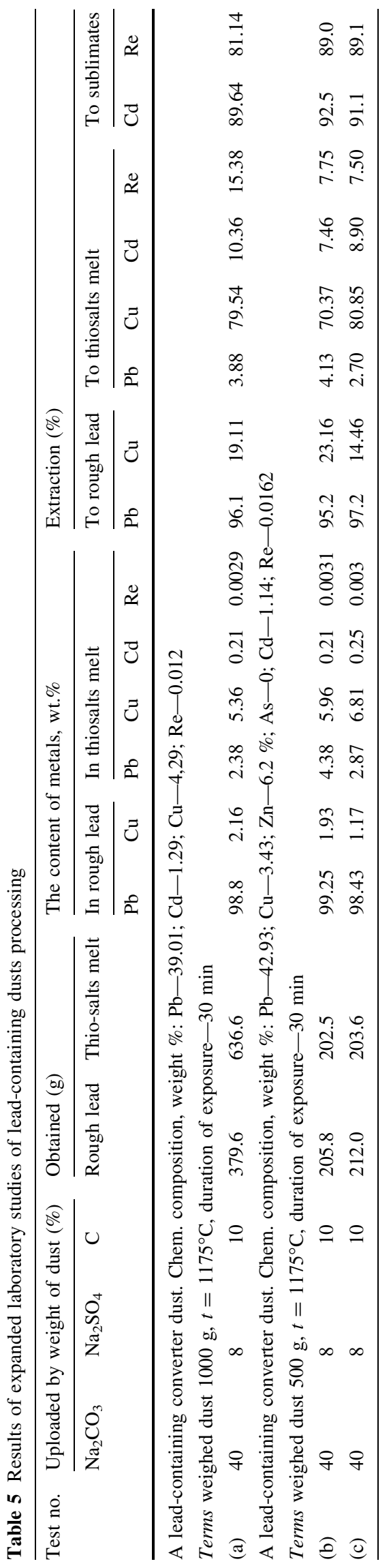

After finding the optimal conditions for processing converter dusts, the scaled-up laboratory investigations were conducted. Experimental conditions and results are shown in Table 4. The table shows that the electrosmelting of lead-containing dusts under reducing medium using optimal conditions from bench-scale causes lead to go into the rough metal up to $97.7 \%$, and copper transition into matte-slag melt at $85.2 \%$. Extraction of cadmium and rhenium to sublimates was 98.1 and $89.8 \%$, respectively. Thus, an improvement was achieved for all four metals under the scaled-up condition giving higher confidence for the development of an industry-level process.

Extraction indices for $\mathrm{Pb}, \mathrm{Cu}, \mathrm{Cd}$, and $\mathrm{Re}$ into the corresponding intermediates under laboratory conditions using a five-factor matrix modeling and scaled-up laboratory studies were mostly identical or better. A flow-sheet has been developed, a shown in Fig. 4, for the developed process. Conducted X-ray analysis (Fig. 5) showed that the values of interplanar spacings of obtained rough lead practically coincide with the tabulated data for the pure lead. Subsequently, rough lead goes to refining by standard techniques. Basic matte-slag melt and secondary sublimates are then processed by hydrometallurgical methods for the recovery of $\mathrm{Cu}, \mathrm{Cd}$, and $\mathrm{Re}$.

Benefits of electrothermal processing of lead-containing dusts include practically complete extraction of lead to the metal at the first stage of electrothermal melting, high recovery of $\mathrm{Re}$ and $\mathrm{Cd}$ into the secondary sublimates and, subsequently to $\mathrm{NH}_{4} \mathrm{ReO}_{4}$ and cadmium sponge as well as the minimization of huge amounts of sulfate lead cakes.

\section{Conclusions}

Experiments on electrothermal processing of lead-containing dusts, using the mathematically modeled test parameters, were performed. The factors of temperature, time, flow rate of soda, sodium sulfate, and a reducing agent (coke) were selected. Separate dependencies, describing the impact of these factors on extraction degree of lead to the metallic phase, copper-to matte-slag melt, cadmium, and rhenium into secondary fumes, were shown. Based on the significant particular dependencies, the compiled multifactor equations were generalized that allowed to calculate the extraction degree of metals to the corresponding intermediates.

The optimal conditions for smelting are as follows: content of $\mathrm{Na}_{2} \mathrm{CO}_{3}-40 \%, \mathrm{Na}_{2} \mathrm{SO}_{4}-8 \%$, and coke-10\% by weight of dust at temperature of $1175{ }^{\circ} \mathrm{C}$ and exposure duration of $30 \mathrm{~min}$. Under these conditions, the highest rates of $\mathrm{Pb}$ extraction to crude metal, $\mathrm{Cu}, \mathrm{Zn}$, and $\mathrm{As}$ to molten sodium thiosalts and $\mathrm{Cd}$ and $\mathrm{Re}$ into secondary fumes were observed. The resulting rough lead is refined 


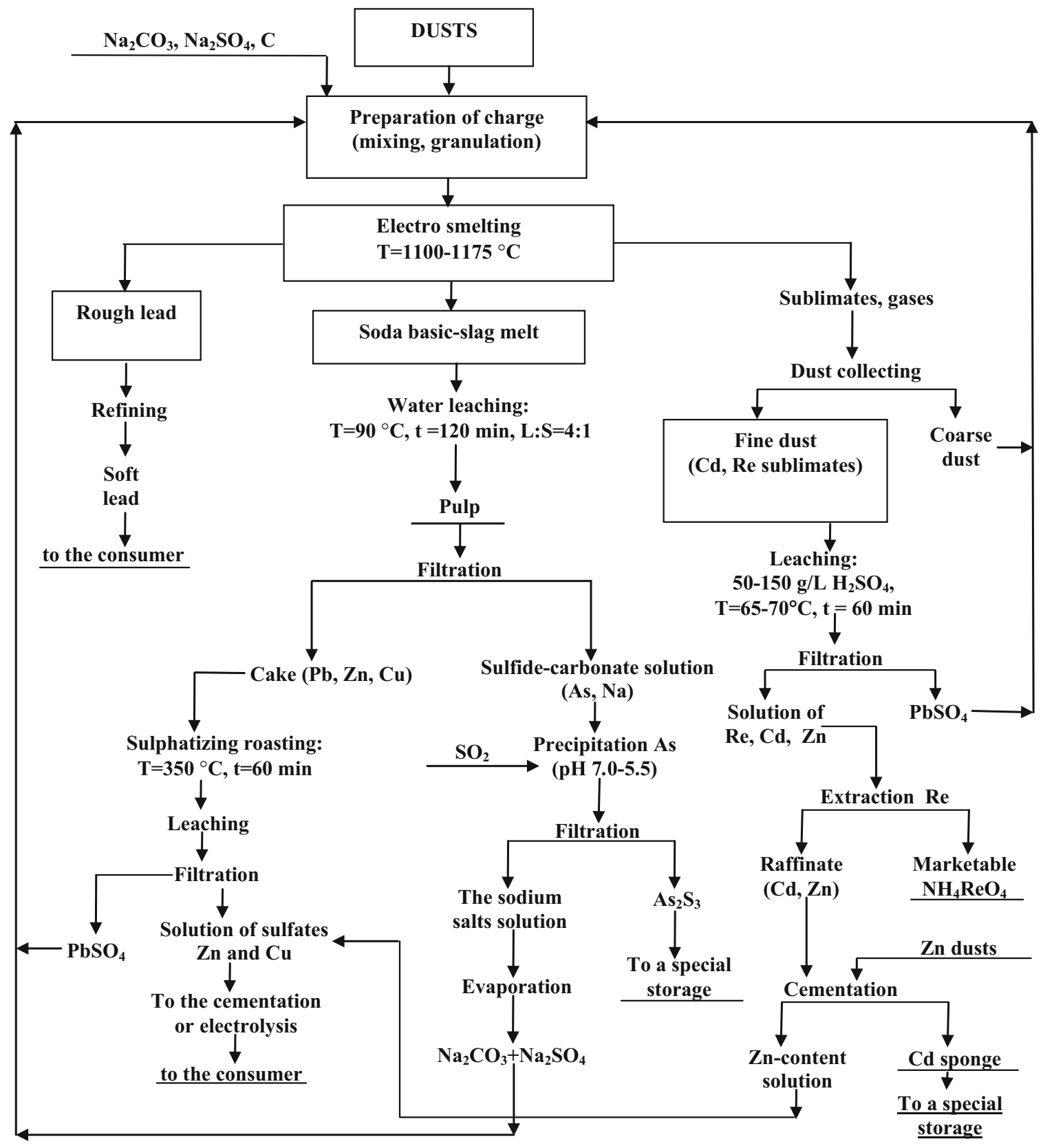

Fig. 4 Flow-sheet for process steps required in the electrosmelting processing of copper-smelter dusts 
Fig. 5 XRD pattern of rough lead obtained after electrosmelting of leadcontaining dusts of copper smelting production

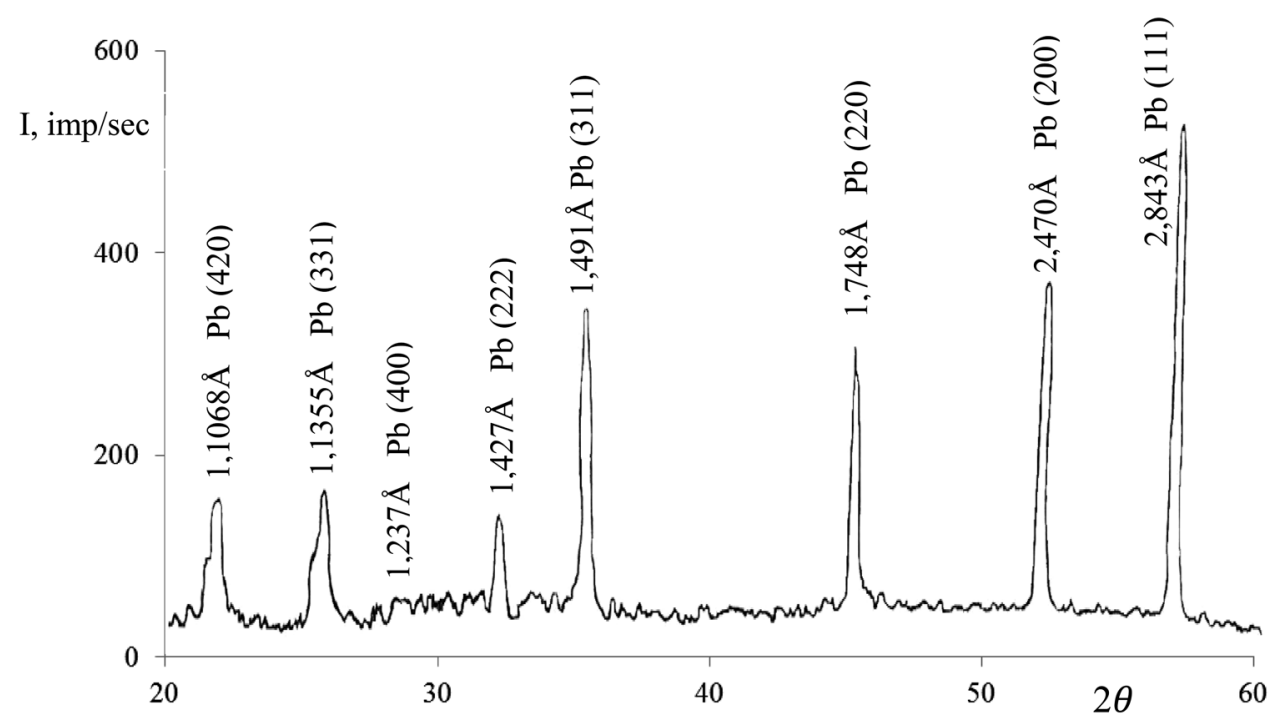

the method of extraction of rhenium from rhenium-containing materials, Publ. 14.11.2003, Bull. No 11

9. Malyshev V, Belyaev SV, Oralov TA, Bukurov TN, Niyazbekov KK (1999) Provisional patent of RK No 8311 C22B 61/00, F27B $1 / 00$, method of roasting a material containing rare elements and the furnace for performing the method, Publ. 15.12.1999, Bull. No 12

10. Belyaev SV, Niyazbekov KK, Solomatina YV, Oralov TA, Baisanov SO, Zharmenov AA, Tolybekov JM (2001) Rhenium extraction from lead and zinc dust of copper smelting production. Complex Use Miner Raw Mater 3-4:40-42

11. Zhalelov RZ, Lata VA, Sokolovskaja LV, Alekseev SO, Kwiatkowski SA, Mazulevskaya TE (2003) Provisional patent of RK No 13156, C22B 7/02. Method for processing of lead dusts, mainly copper production, Publ. 16.06.2003, Bull. No 6

12. Sokolovskaja LV, Alekseev SO, Zhuravlev GM (2001) Sodasulphate melting of lead dusts of copper production. Complex Use Miner Raw Mater 4:64-67

13. Zharmenov AA, Radzhibaev MY, Telbaev SA, Romanov GA, Aisautov MA, Edenbaev SS, Ustemirov HS, Suleimanova GA (2003) Provisional patent of RK No 13567 C22B 61/00, method of processing rhenium-containing lead dusts, Publ. 15.10.2003, Bull. No 10

14. Polyvyanny IR, Kobzhasov AK,Moldabayeva GJ, Polyvyanny VI (2005) Provisional patent of RK No 16461, C22B 7/00, C22B 7/02, C22B 13/00, C22B 61/00, C01G 9/00, C01G 28/00, Method for processing lead cadmium-rhenium-containing converter dusts of copper production, Publ. 15.11.2005, Bull. No 11

15. Malyshev VP (1977) Mathematical planning of metallurgical and chemical experiment, Alma-Ata, Nauka, pp 7-26

16. Protodjakonov MM, Teder RI (1970) Methodology of rational experiments planning. Moscow, Nauka, pp 21-38 